\title{
Tests genéticos: Definición, métodos, validación y utilidad clínica
}

\author{
MARCELA LAGOS L., HELENA POGGI M.
}

Departamento de Laboratorios Clínicos, Facultad de Medicina, Pontificia Universidad Católica de Chile. Santiago de Chile.

\section{Genetic tests: Definition, methods, validity and clinical utility}

The knowledge of the human genome has led to an explosion of available genetic tests for clinical use. The methodologies used in these tests vary widely, allowing the study from chromosomes to the analysis of a single nucleotide. Prior to its use in the clinical setting, these tests should have an evaluation that includes analytical and clinical validation and determination of the clinical utility, as any other tests, including requirements for quality assurance. Recently, the CDC (Centers for Disease Control and Prevention, USA) published a guideline for Good Laboratory Practices for Molecular Genetic Testing for Heritable Diseases and Conditions, covering the preanalytical, analytical and postanalytical phases of the tests. The document covers the importance of proper selection of tests, the availability of information on the performance of the techniques used, the quality control practices, the training of personnel involved and the report of results, to allow the adequate interpretation, including sensitivity and specificity. Considering that recent advances in genetics have changed and will continue to affect clinical practice, genetic tests must meet quality and safety requirements to enable optimal use of them.

(Rev Med Chile 2010; 138: 128-132).

Key words: Clinical Laboratory Techniques; Genetic Techniques; Sensitivity and Specificity.

\section{RESUMEN}

El conocimiento del genoma humano ha dado lugar a un aumento explosivo de los test genéticos disponibles para uso clínico. Las metodologías utilizadas en este tipo de tests son muy variadas, permitiendo desde el estudio de los cromosomas hasta el análisis de una base nucleotídica. Previo a su utilización en el ámbito clínico, estos tests deben tener una evaluación que incluya su validación analítica y clínica $y$ determinación de la utilidad clínica, además de cumplir, como cualquier otro examen, con requisitos para el aseguramiento de la calidad. Recientemente, el CDC (Centers for Disease Control and Prevention, EE.UU) ha publicado recomendaciones para las buenas prácticas de laboratorio de tests moleculares que se utilizan para el diagnóstico de enfermedades genéticas, que abarcan la fase pre-analítica, analítica y post-analítica. Dentro de éstas destacan: la importancia de la selección adecuada de los tests, la disponibilidad de la información sobre el desempeño de las técnicas utilizadas, las prácticas de control de calidad, la capacitación del personal involucrado $y$ la elaboración de un informe de resultados que permita al clínico interpretarlos adecuadamente, incluyendo sensibilidad y especificidad. Tomando en cuenta que los recientes avances en genética han modificado y seguirán modificando la práctica clínica, los test genéticos deben cumplir con las exigencias de calidad y seguridad que permitan su uso óptimo. 


\section{DE LA VIDA REAL}

Paciente femenina de 24 años que consulta por hirsutismo, oligomenorrea y acné, por lo que se indica determinación de niveles plasmáticos de testosterona total y de 17-hidroxiprogesterona basal. Los resultados arrojan valores de testosterona de $100 \mathrm{ng} / \mathrm{dL}$ (valor de referencia: 6-82 $\mathrm{ng} / \mathrm{dL}$ ) y 17-hidroxiprogesterona basal de $3 \mathrm{ng} / \mathrm{mL}$ (valor de referencia 0,2-1,8 $\mathrm{ng} / \mathrm{mL}$ ), por lo cual se solicita 17-hidroxiprogesterona a los $60 \mathrm{~min}$ post estimulación con ACTH, cuyo resultado es de $12 \mathrm{ng} / \mathrm{mL}$. Estos valores sugieren el diagnóstico de hiperplasia suprarrenal congénita por déficit de 21-hidroxilasa, de la forma no-clásica.

¿Qué examen permite confirmar esta sospecha diagnóstica?

Recientes avances en el conocimiento del genoma humano, sobre todo su secuenciación completa, han llevado a un mejor entendimiento del rol de los genes en distintos ámbitos de la biología humana. Como consecuencia, ha aumentado en forma explosiva el número de enfermedades para las que un test genético está disponible; al mismo tiempo se han desarrollado tecnologías que permiten realizar tests altamente específicos, pudiéndose incluso identificar mutaciones de base única. El desafío ha sido trasladar los tests genéticos desde el ámbito de la investigación al uso clínico, para el adecuado manejo de los pacientes.

\section{Definición y clasificación de los tests genéticos}

La definición de "test genético" es extremadamente amplia, sin embargo, la más aceptada actualmente es aquella que incluye "el estudio de ADN, ARN, cromosomas, proteínas y ciertos metabolitos, con el fin de detectar genotipos relacionados a enfermedades, mutaciones, fenotipos o cariotipos con fines clínicos". 1

Los tests genéticos se pueden clasificar de diversas maneras; sin embargo, la clasificación más utilizada se refiere al objetivo del test, por lo que se distingue entre tests diagnósticos y tests predicti$\operatorname{vos}^{2}$. Los primeros son los que permiten confirmar una enfermedad genética sugerida clínicamente; un ejemplo de esto es el estudio molecular del gen FMR1 en un individuo de sexo masculino con retraso mental para el diagnóstico de síndrome de $\mathrm{X}$ frágil. Los tests predictivos, a su vez, se clasifican en dos categorías: los test pre-sintomáticos y los de pre-disposición genética. Los pre-sintomáticos son aquellos que se aplican en individuos en riesgo de sufrir una enfermedad de presentación tardía, como por ejemplo la enfermedad de Huntington; en este caso, un individuo con un test positivo indicaría que el paciente desarrollará la enfermedad en el futuro, pero sin que se pueda precisar cuándo. Los tests genéticos que estudian la predisposición a alguna enfermedad se refieren a tests que implican, en el caso de ser positivos, un mayor riesgo de sufrir una enfermedad en particular, pero sin que se pueda asegurar que van a presentar la enfermedad; un ejemplo es el estudio de los genes BRCA1 y BRCA2 en familias con cáncer de mama-ovario hereditario, en las que la presencia de mutaciones les confiere un riesgo mayor que en la población general de presentar este tipo de cáncer ${ }^{3}$.

Además de estas aplicaciones principales, los tests genéticos pueden ser utilizados con otros fines como, por ejemplo, la prevención de efectos adversos a drogas (farmacogenética), confirmación de enfermedades detectadas por tamizajes, estudio de alteraciones reproductivas, prenatales, así como de paternidad y maternidad, etc. ${ }^{4}$

\section{Estrategias metodológicas en el diagnóstico genético-molecular}

Las metodologías que se utilizan para los tests genéticos son muy variadas, permitiendo desde el estudio de cromosomas hasta el análisis del cambio de una base nucleotídica. Para el estudio de alteraciones del número y estructura de los cromosomas, lo más adecuado será solicitar un estudio citogenético (cariotipo), en cambio, para el estudio de alteraciones más pequeñas, es posible realizar hibridación in situ fluorescente (FISH), la cual incorpora elementos de la biología molecular 
al utilizar sondas marcadas. Frente a una enfermedad en que está descrito el gen responsable, es posible indicar el estudio molecular del gen involucrado, para identificar tanto mutaciones descritas como no descritas.

\section{Requisitos para la implementación de un test genético}

Para poder evaluar si un test genético está listo para el uso clínico, se han reconocido ciertos parámetros. El NIH (National Institutes of Health, EE.UU.), a través del National Human Genome Research Institute, ha publicado un documento para el "Uso seguro y efectivo de los tests genéticos en los Estados Unidos", en que se reconoce la necesidad de evaluar los tests genéticos en un grupo representativo de la población en que se va a aplicar el test, así como la necesidad de entregar recomendaciones para promover el uso seguro y efectivo de estos tests. Este grupo recomienda que los tests sean evaluados respecto de tres parámetros: validación analítica, validación clínica y utilidad clínica, enfatizando además las implicancias éticas, legales y sociales de estos tests ${ }^{1,3,5}$.

\section{Validación analítica}

En el caso de los tests genéticos, la validación analítica se refiere a la exactitud con que el test identifica las mutaciones o el genotipo de interés, e incluye sensibilidad analítica (resultados positivos del test cuando hay una mutación presente), especificidad analítica (resultados negativos del test cuando no hay mutaciones), control de calidad interno y externo, y la evaluación de la "robustez o desempeño" de la técnica.

\section{Validación clínica}

Para un test genético, la validación clínica se refiere a la capacidad del test de diagnosticar o predecir la presencia o ausencia de una enfermedad o condición clínica en particular. Por consecuencia, incluye el que se establezca la sensibilidad y especificidad clínica del test.

Sensibilidad clínica: La sensibilidad clínica se expresa, por lo general, como porcentaje de identificación que se logra con las mutaciones estudiadas cuando está presente la enfermedad. En muchas ocasiones es difícil obtener una sensibilidad clínica adecuada, ya que puede haber más de un gen o región involucrados en el desarrollo de una enfermedad.

Especificidad clínica: La especificidad clínica se refiere al porcentaje de sujetos sin enfermedad cuando la mutación no está presente. Lo que es difícil de interpretar en este caso, son cambios respecto de la secuencia de referencia, que pudiesen no representar un defecto patogénico en el gen estudiado, y corresponder a un polimorfismo (variante normal). Para establecer si se trata o no de la mutación que causa la enfermedad, es de utilidad revisar si la mutación ha sido descrita previamente y realizar el test en ambos padres. Lo ideal, en estos casos, sería hacer estudios en controles y evaluar el efecto de la mutación en la proteína, lo que no siempre es factible para los laboratorios. La especificidad clínica de los tests genéticos también se puede ver comprometida en los casos en que una misma mutación causa distintos fenotipos ${ }^{2}$.

\section{Utilidad clínica}

La utilidad clínica de un test genético se refiere a la utilidad del test en el uso clínico y al valor que tiene la información para la persona en la que se aplica. Se estima evaluando los beneficios y riesgos asociados al uso del test, tomando en cuenta la historia natural de la enfermedad, la disponibilidad y efectividad de las posibles intervenciones en la salud del paciente, así como los costos y la aceptabilidad por parte de los pacientes y médicos ${ }^{3}$.

\section{Estándares de laboratorio para la realización de tests genéticos de uso clínico}

Muchos de los tests genéticos se implementan en un contexto de investigación pero tienen un potencial uso clínico, por lo que ha surgido la necesidad de la aplicación de requisitos para el aseguramiento de la calidad o buenas prácticas para estos tests, los que debieran estar sujetos a los mismos estándares que cualquier otro examen de laboratorio clínico. El CDC ha publicado recientemente recomendaciones para las buenas 
prácticas de laboratorio de los tests moleculares para el diagnóstico de enfermedades genéticas, que abarcan la fase pre-analítica, analítica y postanalítica ${ }^{4}$.

En la fase pre-analítica, el CDC recomienda que el laboratorio entregue información a sus usuarios, incluyendo, al menos, un listado de los tests disponibles, la patología a la cual está dirigido cada test, la región en estudio (gen, mutaciones, etc.), la indicación clínica, la metodología utilizada (en un lenguaje comprensible para el paciente) y las limitaciones del test. Además, deben estar disponibles los formularios de solicitud de exámenes, los consentimientos informados y todo lo concerniente a la toma y transporte de la muestra. El laboratorio, por su parte, requiere para el procesamiento e informe de resultados, que se le entregue información respecto del paciente, la que idealmente debiera incluir: historia clínica relevante, origen étnico e historia familiar atingente (incluido un pedigrí).

En la fase analítica, la recomendación es contar con guías para la validación, la verificación del desempeño de los procedimientos de control de calidad interno y externo, y el tipo de muestra en que se puede realizar el test.

Para la fase post-analítica, se sugiere que el informe de resultado incluya: el método utilizado, especificaciones y limitaciones técnicas, interpretación de los resultados, referencias y recomendaciones de asesoramiento genético (si corresponde), y el correcto uso de la nomenclatura estándar.

\section{Implicancias éticas, legales y sociales}

El diagnóstico de una enfermedad o condición genética no sólo tiene implicancias para el caso índice, sino que también para otros miembros de su familia actual e incluso futura, lo que hace necesario otorgar asesoramiento genético a todos los involucrados 6 .

En los casos en que el resultado del test determine que la causa es genética y heredable surge, además, la posibilidad de discriminación y estigmatización de los pacientes. Esto exige que el laboratorio asegure privacidad a los pacientes, garantizando la confidencialidad de toda la información relacionada con él (o ella) durante todas las fases del proceso. En el caso de tests genéticos, el resguardo de la confidencialidad no es una re- comendación, sino que se exige por ley, también en nuestro país ${ }^{8}$.

\section{Relación entre el médico clínico y el laboratorio}

El progreso en el conocimiento de numerosos nuevos genes y la posibilidad de estudiarlos, ha generado grandes expectativas, pero también gran confusión en torno a la aplicación de estos tests en el ámbito clínico. Para lograr la mayor utilidad clínica de estos tests, es necesaria una estrecha relación entre el médico clínico y el laboratorio, ya que la exactitud del diagnóstico depende de la aplicación del test más adecuado. De la misma forma, el laboratorio debe intentar proveer al clínico una interpretación precisa de los resultados, así como de los datos de sensibilidad y especificidad del test. Para el uso óptimo de los tests, es importante también que los médicos tengan un entrenamiento básico en esta disciplina.

La introducción de la genética y sus métodos diagnósticos ha modificado la práctica clínica, lo que se demuestra, por ejemplo, en el manejo de las familias con cáncer de mama-ovario y colon hereditarios ${ }^{7}$. El uso de estas herramientas también ha sido de gran utilidad en el diagnóstico y la confirmación de enfermedades genéticas, lo que permite, además, un mejor asesoramiento genético a los afectados y sus familias.

En el caso clínico planteado en la "vida real", el estudio genético del gen CYP21A2 identificó la presencia de la mutación Val281Leu en ambos alelos (homocigoto), lo que permitió confirmar la presunción diagnóstica de hiperplasia suprarrenal congénita por déficit de 21-hidroxilasa, de la forma no-clásica.

\section{Referencias}

1. Moore C, Khoury M, Bradley L. From Genetics to Genomics: Using Gene-Based Medicine to Prevent Disease and Promote Health in Children. Semin Perinatol 2005; 29: 135-43.

2. McPherson E. Genetic Diagnosis and testing in Clinical Practice. Clin Med Res 2006; 4: 123-9.

3. Burke W. Clinical Validity and Clinical Utility of Genetic Test. Curr Protoc Hum Genet 2009; Chapter 9: Unit 9.15 . 
4. Chen B, Gagnon M, Shahangian S, Anderson NL, Howerton DA, Boone JD. Centers for Disease Control and Prevention (CDC). Good Laboratory Practices for Molecular Genetic Testing for Heritable Diseases and Conditions. MMWR Recomm Rep. 2009; 58 (RR-6): 1-37; quiz CE-1-4.

5. Holtzman N. Promoting Safe and Effective Genetic test in the United States. Johns Hopkins University Press ISBN-13: 9780801859724
6. Lea DH, Williams J, Donahue MP. Ethical Issues in Genetic Testing. J Midwifery Womens Health. 2005; 50: 234-40.

7. Korf BR. Integration of Genetics into Medical Practice. Growth Horm IGF Res. 2004; 14 Suppl A: S146-9.

8. Ley No 20.120, publicada en el Diario Oficial de 22.09.06. Sobre la investigación científica en el ser humano, su genoma y prohíbe la clonación humana. República de Chile, Ministerio de Salud. 